\title{
Análisis factorial de las características socioeconómicas del ganadero de las cuencas lecheras de la región Amazonas, 2009.
}

\section{Factorial analysis of the socioeconomic characteristics of the watershed dairy farmer in the Amazonas region, 2009.}

\author{
Elías Alberto Torres-Armas ${ }^{1}$, Oscar Andrés Gamarra-Torres ${ }^{2} \&$ Polito Michael Huayama-Sopla $^{3}$
}

\author{
${ }^{1}$ Lic. MsC. En Estadística, Docente Asociado TC., UNTRM Amazonas, eataunas@hotmail.com \\ 2 Biólogo, Docente Auxiliar TC., UNTRM Amazonas, osgat77@yahoo.com \\ ${ }^{3}$ Ing. Agroindustrial, Docente, UNTRM Amazonas, polito michael@terra.com
}

\section{RESUMEN}

El objetivo del presente estudio consistió en la caracterización e identificación de tipologías de ganaderos lecheros de la región Amazonas, con el propósito que estas sirvan para la generación de acciones diferenciadas de trabajo. La metodología aplicada en el presente estudio fue del tipo descriptiva, el método de investigación la encuesta social; la unidad de estudio estuvo compuesta por tres cuencas lecheras, la muestra aleatoria estratificada encuestada fueron 74 ganaderos, distribuidos por asignación proporcional en tres cuencas: Pomacochas, Leymebamba y Molinopampa. En el instrumento de recolección de la información, se consideraron aspectos tecnológicos-productivos lecheros y aspectos sociales. El procesamiento de la información se dividió en dos etapas: para la caracterización se utilizó estadística descriptiva: medias, moda y desviaciones, para la identificación de las tipologías, se recurrió al análisis factorial de correspondencias múltiples. La descripción de los ganaderos lecheros de la región Amazonas, confirmó la heterogeneidad tecnológica-productiva y social, existente entre ellos. El análisis factorial de correspondencias múltiples, permitió distinguir tres tipos de ganaderos, claramente diferenciados por la composición de sus variables.

Palabras clave: análisis factorial, correspondencias múltiples, cuenca lechera.

\section{ABSTRACT}

The objective of the present study was the characterization and identification of tipology of farmers producing milk in dairy watershed of the Amazonas region, with the purpose of that information can be used for the generation of differential actions of work. The methodology applied in the present study was descriptive, and the research method was the social survey. The unit of study was composed by three dairy watershed, the surveyed population was 74 farmers, distributed by proportional allocation in three dairy watershed: Pomacochas, Leymebamba and Molinopampa. The survey collected information related to technological and productive features of dairy production, also some social information was collected. The analysis of the information collected was divided in two stages: for the characterization descriptive statistics were used: means and deviations of the means. For the identification of the tipology, a Factorial Analysis of Multiple Correspondences was used. The description of the dairy producers in dairy watershed of the Amazonas region, confirmed the heterogeneity of technological, productive and social features that coexist among the farmers. The Factorial Analysis of Multiple Correspondences, allowed to distinguish three types of farmers, clearly differentiated by the composition of their variables.

Key words: multiple correspondence factorial analysis, dairy watershed.

INTRODUCCIÓN
En Latinoamérica, las diferentes condiciones socioeconómicas modifican el carácter del agricultor y por tanto, éste no presenta un perfil único ni 
estático, sino una variedad de características cambiantes. No obstante entre estas características existen algunas que lo identifican como sector social: el trabajo familiar sobre la tierra, la posesión de los medios de trabajo, la búsqueda de la reproducción y la relación que sostiene con los mercados. Sin embargo, existen elementos específicos que diferencian al agricultor, por lo cual, se considera importante conocer éstos, para definir estrategias de acción; en el estudio del tipo descriptivo, de investigación social, compuesta por seis centros de acopio lecheros, usuarios del Centro de Gestión Empresarial de Paillaco, con 60 agricultores, distribuidos en tres comunas de la décima región de Chile, donde recolectó información, de aspectos tecnológicos-productivos lecheros y aspectos sociales. Con el análisis factorial de correspondencias múltiples, distinguió tres tipos de agricultores, claramente diferenciados por la composición de sus variables, los cuales deben dar origen a estrategias diferenciadas de trabajo (Guardan y Lerdón, 1998)[1].

Torres et al. (2008) [2], en un estudio sobre la fuente de variación de la productividad del ganado lechero en los distritos de La Florida - Pomacochas (provincia de Bongará), Molinopampa y Leymebamba (provincia de Chachapoyas), región Amazonas; encontraron como factores de variación de la productividad: I. Intensidad de la producción y comercialización, II. Manejo del rebaño, III. Producción bruta utilidad liquida, IV. Ingreso del capital y relación vaca/toro, V. Producción forrajera; concluyendo que el mejoramiento de la productividad económica de las fincas en las cuencas lecheras estudiadas, debe iniciarse fundamentalmente en la búsqueda de mejores niveles de producción por vaca, mejor equilibrio entre las vacas en producción y las de descanso; asimismo una mejora sustancial en el manejo del rebaño; es necesario aumentar considerablemente la productividad de las áreas forrajeras.

La transformación productiva de la agricultura campesina pasa fundamentalmente por la agregación de conocimientos a las personas, sistemas de producción, procesos productivos y a los propios productos. El éxito o fracaso de un negocio se define crecientemente por factores extraprediales y por la forma de entender $y$ articularse con el contexto externo a la unidad productiva; por otra parte, las tecnologías que actualmente hacen más la diferencia entre empresas y países, son las tecnologías "blandas", particularmente de gestión y de diseño de organizaciones (Guardan y Lerdón, 1998) [1]. Por lo tanto, el estudio del campesinado a través del enfoque de tipologías, permite conocer la existencia de una diferenciación social y productiva en el campo, lo cual conlleva a una inserción diferente del proceso productivo y al mejor aprovechamiento de las potencialidades de los distintos tipos de productores que caracterizan al sector campesino; al respecto Defumier (1990) [3], indica que es un error considerar al campesinado como un conjunto homogéneo al que se pueden proponer "paquetes tecnológicos" uniformes. Una tipología, es una forma de conceptualizar dicha realidad, la cual ofrece varios resultados: la organización conceptual de la diversidad existente en la agricultura campesina; la determinación de dominios de recomendaciones; un listado de unidades de producción representativas y las poblaciones de las que es posible hacer inferencias de los resultados (Berdegue, 1991) [4].

Esta situación no es ajena en la gran mayoría de los países de Sudamérica e impone la necesidad de diseñar estrategias de diagnóstico sobre el grado de diferenciación presente en el sector agrícola campesino, para una correcta formulación de políticas diferenciadas de trabajo. Estas políticas para el desarrollo rural deberían partir del reconocimiento de características específicas de diferenciación, considerando además el punto de vista de los campesinos y de sus organizaciones. Sin embargo, en las propuestas de desarrollo rural, escasas veces se ha contado con la participación campesina y la debida consideración de una heterogeneidad social y productiva; por lo que se precisa de la construcción de tipologías de agricultores; basándose en las características socioeconómicas y productivas, que sirvan para analizar sistemáticamente diferencias dentro de cada tipo, las cuales contribuyan a la formulación de políticas diferenciadas de trabajo (Guardan y Lerdón, 1998) [1]; razón por la cual el presente estudio tuvo como propósito la caracterización e identificación de tipologías de agricultores productores lecheros de La Florida-Pomacochas (provincia de Bongará), Molinopampa y Leymebamba (provincia de Chachapoyas) de la región Amazonas, con el propósito que éstas, sirvan para la generación de acciones diferenciadas de trabajo.

\section{MATERIAL Y MÉTODOS}

La población estuvo constituida por todos los ganaderos lecheros de la región Amazonas, Perú. 
2009. La muestra procedente de la población bajo estudio, se seleccionó empleando un muestreo aleatorio estratificado, con afijación proporcional al tamaño del estrato (Santos et al., 2004) [5]; cuyos estratos fueron conformados por las cuencas lecheras de Florida-Pomacochas, Molinopampa y Leymebamba; región Amazonas.

Se emplearon como variables a indicadores sociales como: edad, tenencia de la tierra, aspectos económicos (créditos, subsidios, ahorros, trabajo extrapredial), participación en las organizaciones de la cuenca, condiciones habitacionales, educación y capacitación, salud, mano de obra asalariada, seguridad social; e indicadores tecnológicos productivos como: producción de leche, manejo reproductivo, manejo sanitario, manejo de praderas, alimentación del ganado lechero, manejo higiénico del ordeño, infraestructura predial. Las unidades de muestreo fueron los ganaderos lecheros de la región Amazonas, Perú, 2009; El tamaño de la muestra de productores se determinó con $\mathrm{P}_{h}=0,50$ proporción de productores que poseen la característica de interés en el cuestionario, $Q_{h}=\left(1-P_{h}\right), V=$ varianza esperada dada por $V=(d / z)^{2}=0,002108496, d=9 \%$ precisión ó error máximo de estimación, $z=1,96$ valor de la normal asociado al nivel de confianza del 95\%; obteniendo los siguientes resultados:

$$
n_{0}=\left(\sum w_{h} P_{h} Q_{h}\right) /\left(v+\left(\sum w_{h} P_{h} Q_{h}\right) / N\right)=73,94 \approx 74
$$

ganaderos, a los cuales se evaluaron y encuestaron. De igual manera se realizó la asignación de la muestra con afijación proporcional al tamaño del estrato $_{n_{h}}=W_{h}(n) \quad h=1,2, \ldots, 12$ : Pomacochas 21, Molinopampa 35 y Leymebamba 18.

El diseño de contrastación que se utilizó en la investigación fue el diseño de una sola casilla, correlacional (Hernández, et.al, 2006) [6] y (Tresierra, 2000) [7],

Los datos registrados en el cuestionario de encuesta fueron digitados en una hoja de cálculo del SPSS para Windows y en el paquete estadístico SPADN V 3.25. Los datos se presentan en cuadros y gráficos según las análisis factorial de correspondencias múltiples (Crivisqui, 1994 [8], Benzecri, 1973 [9]).

\begin{tabular}{|c|c|c|c|c|c|}
\hline $\mathbf{N}^{\circ}$ & Indicador & Etiqueta & Modalidades & Cód. & Símbolo \\
\hline \multirow[t]{3}{*}{1} & Edad & EDAD & Joven & 1 & JOVE \\
\hline & & & Adulto & 2 & ADUL \\
\hline & & & Adulto mayor & 3 & ADUM \\
\hline \multirow[t]{6}{*}{2} & & & Sin estudios & 1 & NESI \\
\hline & Nivel de & NE & Inicial & 2 & NEIN \\
\hline & escolaridad & & Primaria & 3 & NEPR \\
\hline & & & Secundaria & 4 & NESE \\
\hline & & & Superior & 5 & NETE \\
\hline & & & $\begin{array}{l}\text { tecnológica/pedagógica } \\
\text { Superior universitaria }\end{array}$ & 6 & NEUN \\
\hline 3 & Participación & PA & Excelente & 4 & PAEX \\
\hline
\end{tabular}

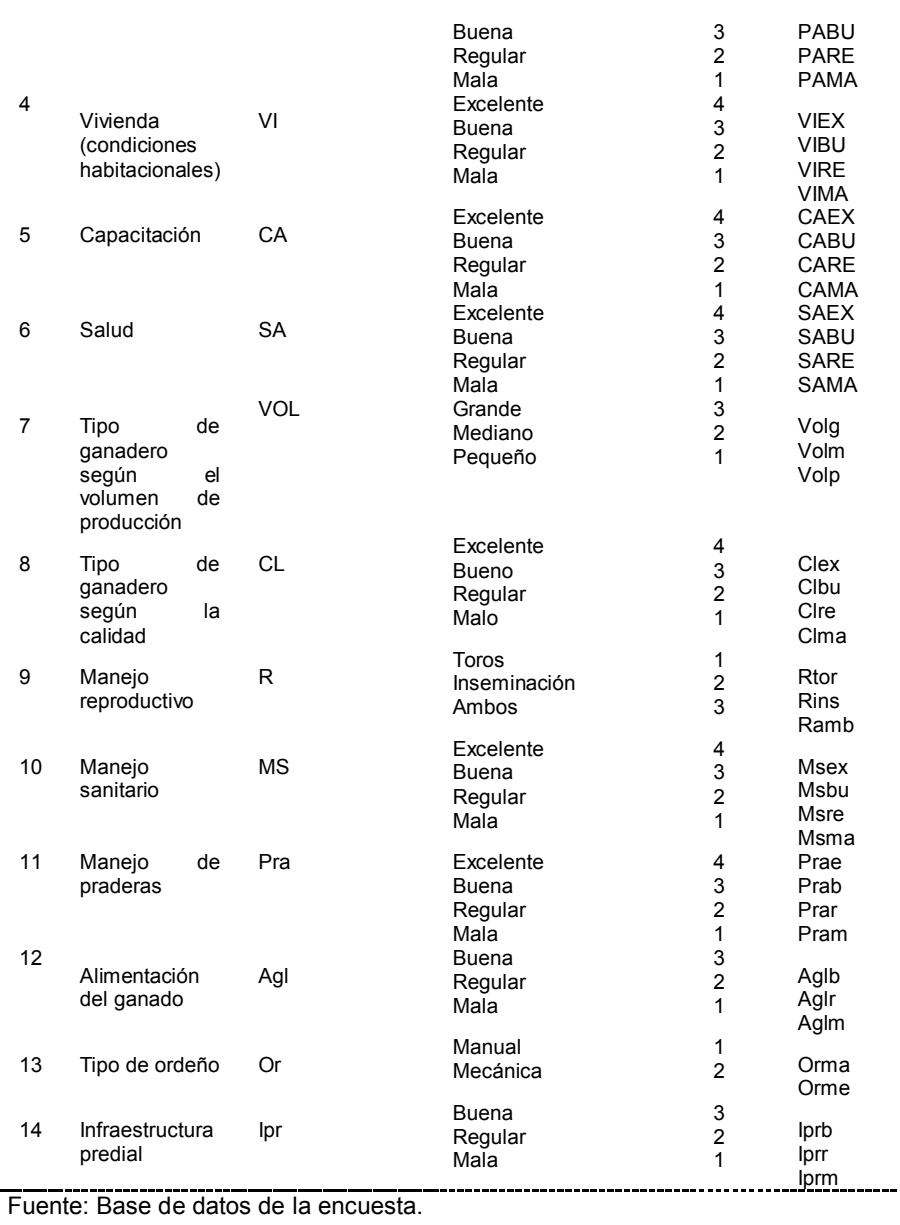

El procesamiento de la información se dividió en dos etapas: la primera, comprendió la caracterización y descripción de los ganaderos, en base a aspectos sociales y tecnológicos-productivos, realizándose análisis cualitativos y cuantitativos de la información; utilizando estadística descriptiva (porcentajes, medias, modas y desviaciones). En la segunda, se identificaron tipologías con el análisis factorial de correspondencias múltiples (AFCM). Para el estudio se consideraron 14 índices mostrados en la Tabla 1, los cuales a su vez estuvieron compuestos por sus respectivas modalidades. El programa computacional utilizado fue el sistema portable de análisis de datos (SPADN) del "Centre Internacional de Statistique et d'Informatique Apliques" (CISIA), Versión 3.1.

\section{RESULTADOS Y DISCUSIÓN}

Para la realización del análisis, se consideraron indicadores sociales como variables nominales ilustrativas (Edad (EDAD), Nivel de escolaridad (NE), Participación (PA), Vivienda (condiciones habitacionales) (VI), Capacitación (CA) y Salud (SA)); las cuales definieron el tema a analizar; y como variables nominales activas, indicadores tecnológicos-productivos, las cuales definen las distancias entre los puntos. La primera etapa del 
ECIPERÚ

análisis consistió en crear tipos de ganaderos en base a indicadores productivos (Tipo de agricultor según el volumen de producción (VOL), Tipo de ganadero según la calidad (CL), Manejo reproductivo (R), Manejo sanitario (MS), Manejo de praderas (Pra), Alimentación del ganado (Agl), Tipo de ordeño (Or), Infraestructura predial (Ipr)); una vez construidos los tipos se procedió a incorporar al análisis los indicadores sociales individualmente.

Los resultados de los valores propios de los porcentajes de inercia, presentados en la Tabla 2, son los obtenidos del análisis factorial de correspondencias, el cual sirvió para la realización de las tipologías.

Tabla 2. Valores propios y porcentajes de inercia de los ganaderos lecheros de la región Amazonas, 2009.

\begin{tabular}{|c|c|c|c|c|c|c|c|c|c|c|}
\hline & F1 & F2 & F3 & F4 & F5 & F6 & F7 & F8 & F9 & F10 \\
\hline Valor propio & 0,251 & 0,188 & 0,156 & 0,150 & 0,136 & 0,130 & 0,126 & 0,119 & 0,105 & 0,097 \\
\hline Inercia (\%) & 9,486 & 7,115 & 5,917 & 5,670 & 5,147 & 4,938 & 4,751 & 4,490 & 3,967 & 3,660 \\
\hline$\%$ acumulado & 9,486 & 16,601 & 22,518 & 28,189 & 33,336 & 38,274 & 43,025 & 47,515 & 51,481 & 55,142 \\
\hline Inercia ajustada & 0,037 & 0,016 & 0,008 & 0,007 & 0,005 & 0,004 & 0,003 & 0,003 & 0,001 & 0,001 \\
\hline Inercia ajustada (\%) & 30,512 & 12,908 & 6,850 & 5,840 & 3,962 & 3,312 & 2,783 & 2,118 & 1,059 & 0,608 \\
\hline$\%$ acumulado & 30,512 & 43,420 & 50,270 & 56,110 & 60,073 & 63,385 & 66,168 & 68,285 & 69,345 & 69,952 \\
\hline
\end{tabular}

En la Tabla 2, se observa el decrecimiento relativo de los valores propios. La inercia captada por los ejes 1 y 2 indica su importancia en las tipologías obtenidas, el estudio de estos resultados permitió detectar las variables que más contribuyeron en la formación de los ejes 1 y 2 , y a posterior, en la tipificación o en la diferenciación de las estructuras de producción.

El análisis factorial de correspondencias aplicado permitió identificar tres tipos principales constituidos por bloques de individuos bastante próximos, de acuerdo a las 48 modalidades de las variables utilizadas. El análisis factorial fue interpretado con la ayuda de los resultados obtenidos en la construcción de grupos obtenidos sobre los factores 1 y 2 de las modalidades (Tabla 3 ), donde se muestra la identificación de la modalidad.

En el TIPO I, las variables que más aportaron en la calificación del ganadero fueron: posee un mal manejo de praderas (Pram), sin embargo, el manejo reproductivo se hace ambas formas ya sea usando toros o inseminación artificial, (Ramb) y su manejo sanitario oscila entre regular (Msre) y excelente (Msex), por el sistema de vacunación como por los tratamientos antiparasitarios y la asistencia veterinaria programada.

En el TIPO II, las variables que más aportan a la calificación de estos ganaderos fueron: el tipo regular de ganadero según la calidad de la leche (Clre), además su manejo sanitario es malo (Msma) y la alimentación del ganado lechero es malo (Aglm) $y$ regular (Aglr), no conservan forraje $y$ no suministran concentrado.

Tabla 3: Grupos construidos sobre el factor 1 y 2 de las modalidades según los indicadores tecnológicos productivos de los ganaderos lecheros de la región Amazonas, 2009.

\begin{tabular}{|c|c|c|}
\hline ID. & MODALIDAD & VARIABLE \\
\hline \multicolumn{3}{|c|}{ TIPO I } \\
\hline Pram & Mala & Manejo de praderas \\
\hline Msre & Regular & Manejo sanitario \\
\hline Ramb & Ambos & Manejo reproductivo \\
\hline Msex & Excelente & Manejo sanitario \\
\hline \multicolumn{3}{|c|}{ TIPO II } \\
\hline Aglm & Mala & Alimentación del ganado \\
\hline Msma & Mala & Manejo sanitario \\
\hline Clre & Regular & Tipo de agricultor según la calidad \\
\hline Aglr & Regular & Alimentación del ganado \\
\hline \multicolumn{3}{|c|}{ TIPO III } \\
\hline Rtor & Toros & Manejo reproductivo \\
\hline Prar & Regular & Manejo de praderas \\
\hline Msbu & Buena & Manejo sanitario \\
\hline Rins & Inseminación & Manejo reproductivo \\
\hline
\end{tabular}

EI TIPO III, se caracteriza por que las variables que más aportan fueron: ganaderos según el manejo regular de praderas (Prar), en el encaste, hay quienes usan toros (Rtor) y otros que usan inseminación (Rins), este grupo empieza a perder el temor por el uso de la inseminación artificial; su manejo sanitario es bueno (Mabu). A las tipologías productivas ya constituidas, se incluyeron en forma individual los indicadores sociales anteriormente descritos, para poder determinar la influencia de dichos indicadores en estos tres grupos o tipos de ganaderos (Tabla 4).

\begin{tabular}{|c|c|c|}
\hline ID. & MODALIDAD & VARIABLE \\
\hline $\begin{array}{l}\text { SAMA } \\
\text { ADUM } \\
\text { SAEX } \\
\text { VIEX }\end{array}$ & \begin{tabular}{l}
\multicolumn{1}{c}{ TIPO I } \\
Mala \\
Adulto mayor \\
Excelente \\
Excelente \\
TIPO II
\end{tabular} & $\begin{array}{l}\text { Salud } \\
\text { Edad } \\
\text { Salud } \\
\text { Vivienda }\end{array}$ \\
\hline
\end{tabular}




\begin{tabular}{lll} 
NEIN & Inicial & Nivel de escolaridad \\
SABU & Buena & Salud \\
SAMA & Mala & \\
& Salud \\
PABU & TIPO III & \\
PARE & Buena & $\begin{array}{l}\text { Participación } \\
\text { Pegular }\end{array}$ \\
\hline
\end{tabular}

Dentro el TIPO I se encuentran ganaderos adultos mayores (ADUM), los cuales según la clasificación utilizada en el presente estudio son personas que se encuentran con más 60 años de edad, con previsiones de salud mala (SAMA), y excelente (SAEX); y la situación habitacional excelente (VIEX).

Para el TIPO II, muestra a ganaderos cuya característica respecto al nivel de escolaridad es que tienen nivel inicial (NEIN) y respecto a la previsión tanto para la salud como para la jubilación es mala (SAMA) y buena (SABU). Esta característica respecto a la variable vivienda es regular (VIRE) y mala (VIMA); la capacitación es mala (CAMA); es regular (SARE).

EI TIPO III, muestra a ganaderos cuyas características respecto a la participación es regular (PARE) y buena (PABU).

\section{DISCUSIÓN}

Ahumada (1996) [10] y Rojas y Reyes (1987) [11], usando la edad de los agricultores como un elemento de tipificación en trabajos realizados en la zona central de Chile, encontraron que el promedio de edad fluctuaba entre los 32 y 61 años. Estos datos son similares a los encontrados en este trabajo de investigación cuya edad de los productores lecheros fluctuaba entre 26 y 82 años, con un promedio de 49 años.

En la actualidad INDAP (1996) [12], propone que la participación en las organizaciones no solo debe darse en la elección de representantes si no que éstas deben ser consideradas como espacios donde plantear problemas, participando también en la búsqueda de soluciones a ellos. Al respecto CIDER (1996)[13], propone la incorporación institucionalizada, en los distintos proyectos de desarrollo rural, de la población usuaria. Gómez (1996) [14], señala que las organizaciones, ya sean sociales o productivas, son elementos que por su influencia cada vez más importante en la gestión socio-productiva, despiertan creciente interés en el habitante rural e incrementan los niveles de participación, lo cual también ha sido confirmado en la presente investigación.
Guardan y Lerdón (1998) [1], señala que los campesinos viven con intensidad particular, las carencias de agua potable, la calidad de la vivienda, las condiciones de habitabilidad (hacinamiento de tres o más personas por pieza) y el acceso a alguna fuente de energía. En este estudio, se encontró viviendas construidas en un área promedio de $147,13 \mathrm{~m}^{2}$.

El Censo de 2007 (INEI, 2007) [15], indica que el promedio de personas por familia a nivel nacional es de 4,7 y en el área de selva rural es de 5,5; cuyo valor si bien no es alto tiene una influencia negativa en el ingreso percápita (MIDEPLAN, 1995) [16]. Así mismo, en número promedio de habitaciones es de 6,75 habitaciones por vivienda, pudiendo existir entre 2 a 15 habitaciones; resultados similares al que se encontró en este estudio, un promedio de 4,68 personas por vivienda.

En educación y capacitación, los resultados son muy parecidos a los resultados de Morandes (1993) [17], quien indica que el nivel educacional de los agricultores es un factor limitante, dentro de un rango que va desde el analfabetismo hasta niveles variados de educación primaria y algunos grados de secundaria; asimismo Ahumada (1996) [10], encontró en la región del Maule que un $48 \%$ de sus encuestados tenía un nivel de estudios básicos, porcentaje similar al determinado en esta investigación.

De este modo, la educación y la capacitación en los medios rurales enfrentan el desafío de resolver este problema, es decir, lograr desarrollar efectivamente los aprendizajes para un mundo cambiante, sin invalidar los marcos de significados de la tradición local, de forma que la población rural pueda, por una parte, satisfacer sus necesidades humanas en el contexto de las culturas locales y, por otra, articularse con la dinámica de la sociedad global. Se encontró que el agricultor participa y hace participar en capacitación a las personas involucradas en su producción lechera, lo que concuerda con Lacki (1995) [18], que indica que es imprescindible capacitar a todos los miembros de las familias rurales, con el propósito de liberar y desarrollar un inmenso potencial latente, con el fin de que estén en efectivas condiciones de introducir innovaciones tecnológicas, gerenciales y organizativas.

El resultado de la mano de obra asalariada (50\%), es porcentualmente mayor a lo encontrado por Ahumada (1996) [9] en la región del Maule, quien encontró que el $37 \%$ de las familias estudiadas incorpora mano de obra asalariada en sus labores productivas, lo que indica que utilizan principalmente 
mano de obra familiar; es decir, que los pequeños productores utilizan básicamente los recursos de fuerza de trabajo que proveen los miembros de la familia, que pertenecen al hogar.

Las necesidades de salud, se vinculan a los bienes y servicios que habría que proporcionar a la población, prescindiendo de los deseos o exigencias de las personas, las dificultades de acceso 0 las condiciones económicas de los individuos, para asegurar a todos, protección y atención médica adecuada (Arcos, 1988) [119]; sin embargo, se encontró un alto porcentaje que no hace previsión para su salud y jubilación. CIDER (1996) [13], señala que la previsión afecta a una gran parte de la población rural, especialmente a los pequeños productores, y como un medio de modernizar la vida rural, indica que se debería considerar una modalidad específica de cotizaciones, como ejemplo, sugiere el pago de cotizaciones que contemple tres o cuatro cuotas periódicas anuales y no la cotización mensual, pero que garantice una prestación de servicio permanente por parte del sistema.

\section{CONCLUSIONES}

El análisis factorial de correspondencias múltiples, realizado para las variables tecnológicas-productivas permitió identificar tres tipos de ganaderos:

TIPO 1, Se caracteriza por las variables que más aportan en la calificación del ganadero lechero son: que posee un mal manejo de praderas, sin embargo, el manejo reproductivo se hace en ambas formas, ya sea usando toros e inseminación artificial, es decir, en el encaste se usan ambas técnicas como ser toros e inseminación; su manejo sanitario oscila entre regular) y excelente, por el sistema de vacunación como por los tratamientos antiparasitarios y la asistencia veterinaria programada.

En el TIPO 2, las variables que más aportan a la calificación de estos ganadores son: el tipo regular de ganadero según la calidad de la leche, además su manejo sanitario es malo y la alimentación del ganado lechero es malo y regular, no conservan forraje y no suministran concentrado.

EI TIPO 3, se caracteriza por que las variables que más aportan son: ganaderos según el manejo regular de praderas, en el encaste, hay quienes usan toros y otros que usan inseminación, este grupo empieza a perder el temor por el uso de la inseminación artificial; su manejo sanitario es bueno.

\section{REFERENCIAS}

[1] Guardan, D. \& J. Lerdón. 1998. Caracterización y tipificación de agricultores usuarios del Centro de Gestión Empresarial de Paillaco. Facultad de Ciencias Agrarias Universidad Austral de Chile Casilla 567. Valdivia. Chile. Disponible en:

http:/ / mingaonline.uach.cl/scielo.php?script=sci_artte xt\&pid=S030480219990002 00008\&lng=es\&nrm=iso.

[2] Torres, E.; O. Gamarra \& P. Huayama. 2008. Análisis de la productividad lechera en las cuencas lecheras de la región Amazonas, Perú. 2008. Informe final de investigación. Universidad Nacional Toribio de Mendoza de Amazonas. Carrera Profesional de Ingeniería Agroindustrial. 45 pp.

[3] Defumier, M. 1990. Importancia de la tipología de unidades de producción agrícola en el análisis de diagnóstico de realidades agrarias. Ed. Red Internacional de Metodología de Investigación de Sistemas de Producción. Santiago, Chile. 63-82 pp.

[4] Berdegue, J. 1991. Efectos de la metodología de tipificación en la investigación de sistemas de producción. Ed. Red Internacional de Metodología de Investigación de Sistemas de Producción (RIMISP). Santiago, Chile. 251 -265 pp) 175-185.

[5] Santos, J.; A. Muñoz; P. Juez \& P. Cortiñas. 2004. Diseño de encuestas para estudios de mercado. Primera reimpresión. Editorial Centro de Estudios Ramón Areces, S.A. 167-183 pp.

[6] Hernández, R.; C. Fermandez \& P. Baptista. 2006. Metodología de investigación. 4ta. Edición. Editorial McGrawHill. México. 208-212 pp.

[7] Tresierra, A. 2000. Metodología de investigación. $4^{\text {ta }}$. Edición. Editorial Biociencia. Trujillo. 81 pp.

[8] Crivisqui, E. \& G., Villamonte. 1994. Presentación de los métodos estadísticos de análisis factorial de correspondencias simples y múltiples. Universidad Nacional de Trujillo - Perú. Université Libre de Bruxelles. PRESTA. 105 pp.

[9] Benzecri, J.P. 1973. Lanalyse des donnés. L'analyse des correspondances, Dunod, v 2, París. 9-40 pp.

[10] Ahumada, M. 1996. Estudio de la racionalidad de la economía campesina en la localidades de La Calor, Talca Chico, Tabunco y Putu, de la provincia de Talca, VII región de Chile. Tesis de Maestría en desarrollo rural. Valdivia. Universidad Austral de Chile, Facultad de Ciencias Agrarias. 101 pp.

[11] Rojas, A. y J. Reyes. 1987. Diferenciación de los productores familiares campesinos en el Chile Central. Talca, Chile. Fundación O.C.A.C. 106 pp.

[12] Instituto de Desarrollo Agropecuario (INDAP). 1996. Memoria. Santiago, Chile. 88 pp.

[13] Comisión Interministerial para el Desarrollo Rural (CIDER). 1996. Modernización de la vida rural. Santiago. 185 pp.

[14] Gómez, S. 1996. Marco teórico metodológico para el 
análisis de las organizaciones rurales en Chile. Curso Seminario, Desarrollo rural sustentable y gestión de empresas agrícolas. Valdivia. 218 pp.

[15] Instituto Nacional de Estadística e Informática (INEI). 2007. Niveles de pobreza. Disponible en: http://www1.inei.gob.pe/biblioineipub/bancopub/Es t/Lib0180/C8-1.HTM. Visitado 12-12-2009.

[16] (MIDEPLAN). 1995. Apuntes metodológicos para la elaboración de estrategias y planes regionales. Santiago, Chile. 11-14 pp.

[17] Morandes, A. 1993. Evaluación de la adopción de tecnología. CEDRA. Santiago, Chile. 95 pp.

[18] Lacki, P. 1995. Desarrollo agropecuario: de la dependencia al protagonismo del agricultor. Serie Desarrollo rural No 94 ta. Edición. Santiago, 145 pp.

Ministerio de Planificación y Cooperación

[19] Arcos, M.E. 1988. Elaboración y aplicación de un modelo de evaluación de calidad de vida en una comunidad rural. Tesis de Maestría en desarrollo rural. Valdivia. Universidad Austral de Chile, Facultad de Ciencias Agrarias. 157 pp.

\section{E-mail: allpachaki@hotmail.com}

